\title{
1-Year Evaluation of OT Bridge Abutments for Immediately Loaded Maxillary Fixed Restorations: A Multicenter Study
}

\author{
Rosario Acampora ${ }^{1}$ Marco Montanari ${ }^{1}$ Roberto Scrascia ${ }^{1}$ Emiliano Ferrari ${ }^{1}$ Massimo Pasi ${ }^{2}$ \\ Gabriele Cervino $^{3}$ Silvio Mario Meloni ${ }^{4}$ Aurea Immacolata Lumbau ${ }^{4}$ Erta Xhanari ${ }^{5}$ \\ Alba Koshovari ${ }^{5}$ Marco Tallarico ${ }^{6}$
}

${ }^{1}$ Private Practice, Italy

2Department of Dental, CLMOPD Ateneo Vita-Salute San Raffaele, Milan, Italy

${ }^{3}$ Department of Biomedical and Dental Sciences, Morphological and Functional Images, School of Dentistry, University of Messina, Policlinico G. Martino, Via Consolare Valeria, Messina, Italy

${ }^{4}$ Department of Medical, Surgical and Experimental Sciences, University of Sassari, Sassari, Italy

${ }^{5}$ Departament of Implantology and Prosthetic Aspects, Master of Science in Dentistry Program, Aldent University, Tirana, Albania

${ }^{6}$ School of Dentistry, University of Sassari, Sassari, Italy
Address for correspondence Marco Tallarico, DDS, MSc, School of Dentistry, University of Sassari, 07100 Sassari, Italy (e-mail: me@studiomarcotallarico.it).

\begin{abstract}
Keywords

- OT Bridge

- dental implants

- immediate loading

- complete edentulous patients

- maxilla
\end{abstract}

Objective Preliminary data on survival and success rates of immediately loaded, maxillary, screw-retained, implant-supported, fixed restorations delivered on narrow and low-profile OT Equator abutments (OT Bridge, Rhein'83) were evaluated.

Materials and Methods This retrospective study evaluated data collected from patients rehabilitated with OT Bridge prosthetic concept between November 2017 and February 2019 in six different centers. Outcome measures were implant and prosthetic survival rates, biological and technical complications, marginal bone loss (MBL), oral health impact profile (OHIP), bleeding on probing, and plaque index.

Results A total of 76 implants were inserted in 14 patients. Patients were followed for a mean period of 15.8 months (range $=12-24$ ). All the patients receive OT Equator (Rhein'83) as intermediate abutments. One year after loading, one implant failed $(1.3 \%)$. None of the prosthesis failed. One prosthetic complication was experienced in one patient. Three out of 76 implants were connected to the prosthetic framework using only the Seeger system, without screw. Difference in OHIP values was statistically significant $(71.9 \pm 8.5 ; p=0.000)$. One year after loading, MBL was $0.21 \pm 0.11 \mathrm{~mm}$ and $p$-value was 0.000 . One year after loading, $8.7 \%$ of the examined implant sites present positive bleeding on probing, while $6.4 \%$ of the implant sites presented plaque.

Conclusion The OT Equator abutments (Rhein'83) showed successful results when used to support maxillary fixed dental prosthesis delivered on four to six implants. High implant and prosthetic survival rates, very low complications, high patient satisfaction, and good biological parameters, including only $0.2 \mathrm{~mm}$ of bone remodeling were experienced one year after function. Further studies are needed to confirm these preliminary results.
DOI https://doi.org/

$10.1055 / \mathrm{s}-0040-1716632$

ISSN 1305-7456.
(C) 2021. European Journal of Dentistry.

This is an open access article published by Thieme under the terms of the Creative Commons Attribution License, permitting unrestricted use, distribution, and reproduction so long as the original work is properly cited. (https:// creativecommons.org/licenses/by/4.0/)

Thieme Medical and Scientific Publishers Pvt. Ltd., A-12, 2nd Floor, Sector 2, Noida-201301 UP, India 


\section{Introduction}

Nowadays dental implants are widely used to overcome edentulism. In patients requiring a complete rehabilitation different number of implants may be installed. Although there is a continuous debate on the most appropriate implant number and position, six implants seem to be preferable to four in the medium term follow-up. ${ }^{1}$ Then patient can receive immediate or delayed loading protocol according to the well-established criteria, such us primary implant stability. ${ }^{2,3}$ Following this protocol, patients may receive definitive abutment at the time of surgery, potentially reducing the amount of bone loss. ${ }^{4,5}$ Different abutments can be used to deliver a temporary or definitive implant-supported restoration. According to the clinician's preference, the restoration could be cemented- or screw-retained. ${ }^{6}$ There are no clear benefits of one over the other one. In 2018, Tallarico et al conduced a consensus conference on prosthetic aspect showed that screw-retained restorations should be suggested because they are easier to be removed. ${ }^{6}$ The main concern regarding implant-supported and screw-retained restorations is that the implants shall be as much parallel as possible. It is not easy to evaluate the maximum degree of accepted disparallelism between implants. For internal conical connection, the maximum degree that could be accepted is the double of the internal degree of the tapered connection. This concept derives from the bisector theorem. Nevertheless, the overall number of placed implants and their tridimensional position make it difficult to be established. Computer-assisted and template-based surgery may allow for parallel implants even if errors in angle may occur..$^{7-9}$ To overcome this drawback, the conversion of internal conical connections is mandatory to ensure accurate fitting of the prosthetic frameworks. According to a recent systematic review, misfit and microleakage within the implant-abutment interface may create biological and technical problems, favoring the onset of peri-implant diseases. ${ }^{10}$ At today, multiunit abutments are widely used to convert the implant connections, allowing for an abutment level restoration. In addition to conversion of the platform, an abutment-level restoration reduces soft tissue inflammation, patient discomfort, and finally allow to reduce peri-implant marginal bone loss (MBL). In the recent years, a narrow and low-profile abutment has been proposed for screw-retained and implant-support restorations delivered at abutment level. The same abutments were already successful used in dentistry to retain implant overdentures. ${ }^{11-15}$

Mechanical features of low-profile prosthetic retention devices during chewing cycles were analyzed in several in vitro studies by applying finite element method (FEM) and Von Mises analyses. ${ }^{16-20}$ These researches concluded that low-profile abutments offers long-term success when applied following the manufacture guidelines. A possible explanation of this result was found in two of these studies. ${ }^{19,20}$ The shape of the OT Equator abutment (Rhein'83) seems to distribute the strength over the head of the retainer, allowing the higher stress on the retainer gum. This characteristic involves minor stress around the fixture-abutment complex and the peri-implant bone tissue. ${ }^{19,20}$
The aim of the present multicenter study is to report preliminary retrospective data on survival and success rates of immediately loaded, maxillary, screw-retained, and implant-supported restorations delivered on narrow and low-profile OT Equator abutments (Rhein'83) according to the OT Bridge prosthetic protocol.

\section{Materials and Methods}

This study was designed as retrospective case series study. A chart review of previously treated patients received immediately loaded, maxillary, screw-retained, implant-supported restorations, and delivered on narrow and low-profile OT Equator abutments (Rhein'83, Bologna, Italy) were performed by an independent examiner. All the treatments were performed between November 2017 and February 2019. A written informed consent for surgical and prosthetic procedures including clinical and radiological data were obtained from each patient. This research adhered to the principles embodied in the Declaration of Helsinki of 2013. Medical data were anonymized so that patient cannot be identified. The study protocol was approved by the ethics committee of Aldent University in Tirana (Protocol n ${ }^{\circ} 4 / 2020$ ).

After accurate evaluation of available documents, radiographs, and pictures, all the screened patients were included or excluded according to the following criteria.

The inclusion criteria were:

- Aged 18 years or older

- Able to sign an informed consent

- Immediately loaded restorations

- Maxillary arch

- Screw-retained prosthesis

- OT Equator abutments

- 1 year after loading follow-up

The exclusion criteria were:

- General contraindications for oral surgery

- Heavy smokers ( $\geq 11$ cigarettes per day)

- Poor oral hygiene defined as bleeding on probing index $>25 \%$

- Local acute or chronic infections

- History of substance abuse (drugs or alcohol) and/or psychiatric problems

- Pregnancy or lactation

- Current or past treatment with intravenous bisphosphonates

- Irradiation of the neck or head area in the past 5 years

- Absence of teeth in the opposite jaw

- Severe bruxism or jaw clenching

All the implants were placed according to the manufacturer instructions. Immediately after implant placement, narrow- and low-profile OT Equator abutments (Rhein'83) were screwed to the implants according to the manufacturer and in agreement with the one-abutment at one-time concept (-Fig. 1). Afterward, cylindric "extragrade" abutments (Rhein'83) were screwed onto the OT Equators (Rhein'83), and the prefabricated acrylic temporary restoration rebased (-Fig. 2). After implant placement and loading, all the 

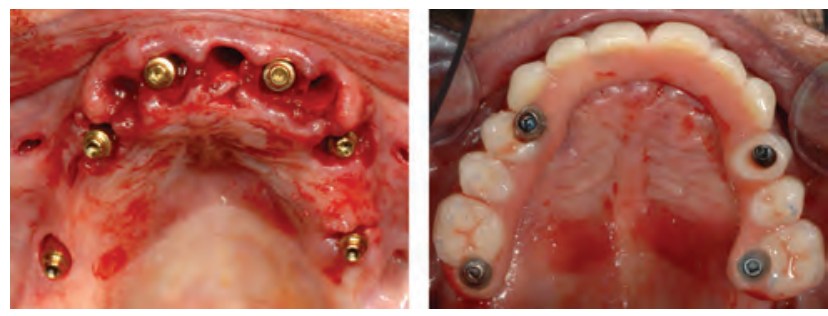

Fig. 1 Intraoral view immediately after implant placement and loading.

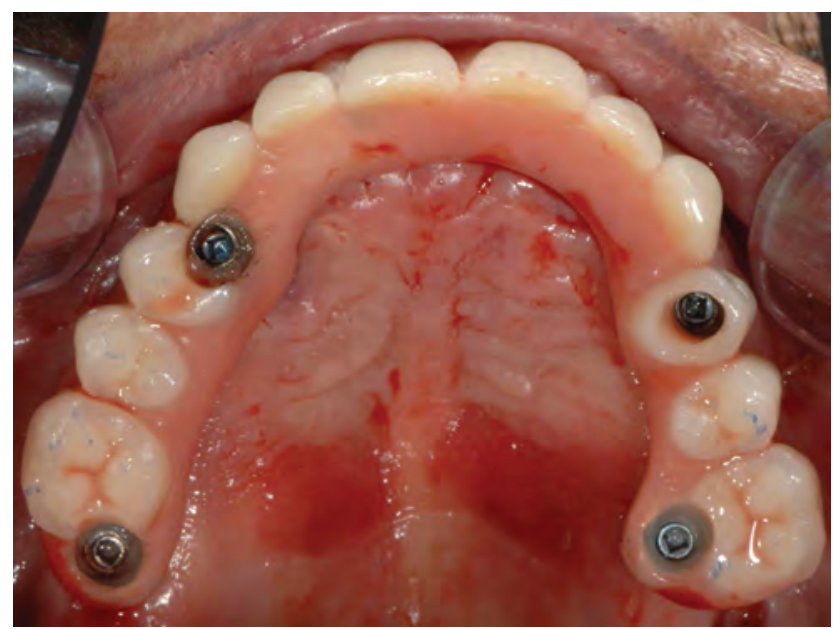

Fig. 2 Intraoral occlusal view at immediate loading (enlargement).

patients received drugs prescription and oral and written recommendations about the correct oral hygiene maintenance and diet. Total 3 to 5 months after implant placement, definitive, abutment-level impressions were taken and screw-retained, CAD/CAM, titanium-composite restorations

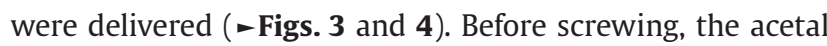
rings (Seeger system, Rhein'83) were placed between the subequatorial area of the OT Equators (Rhein'83) and the cylindrical "extragrade" framework connections. The prosthesis was screwed twice at $20 \mathrm{Ncm}$. Occlusion was adjusted and patients were enrolled in a strictly follow-up protocol including occlusal adjustment and hygiene maintenance every 6 months and radiographs every year (-Fig. 5).

\section{Outcomes Measures}

- Implant failure was defined as mobility, infection, fracture, and/or any other mechanical or biological issue that determined its removal.

- A prosthesis was considered a failure any time it had to be replaced.

- Any biological (e.g., drug-resistant pain, swelling, excessive MBL, suppuration, etc.) and/or technical (e.g., fracture of the veneering material and or framework, screw loosening, etc.) complications were recorded during follow-up.

- Marginal bone levels measured as the distance between the implant platform to the first bone to implant contact. Mean values of mesial and distal measurements were calculated for each implant. Marginal bone level was measured on digital periapical radiographic images obtained by parallel

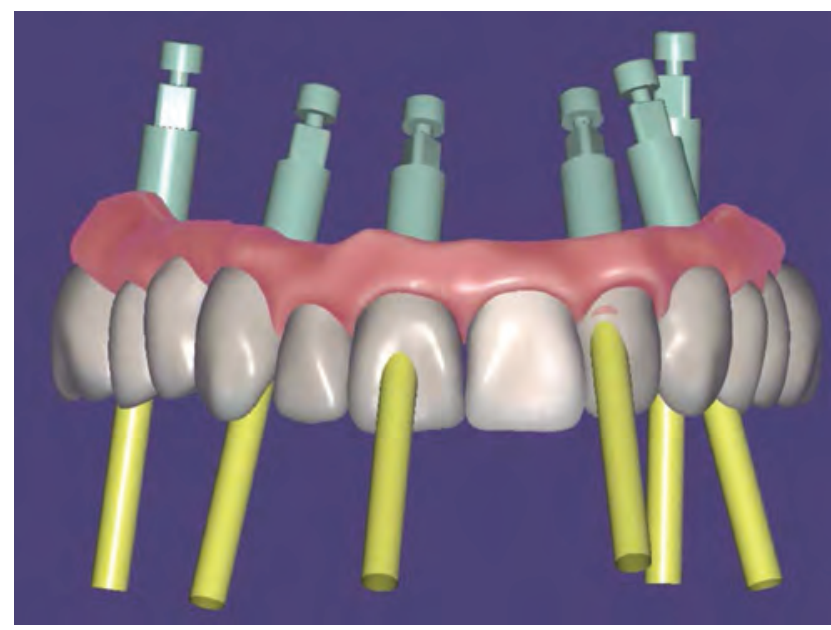

Fig. 3 Computer aided design of the definitive prosthesis. The two anterior implants were leaved only with the Seeger system avoiding the prosthetic screw.

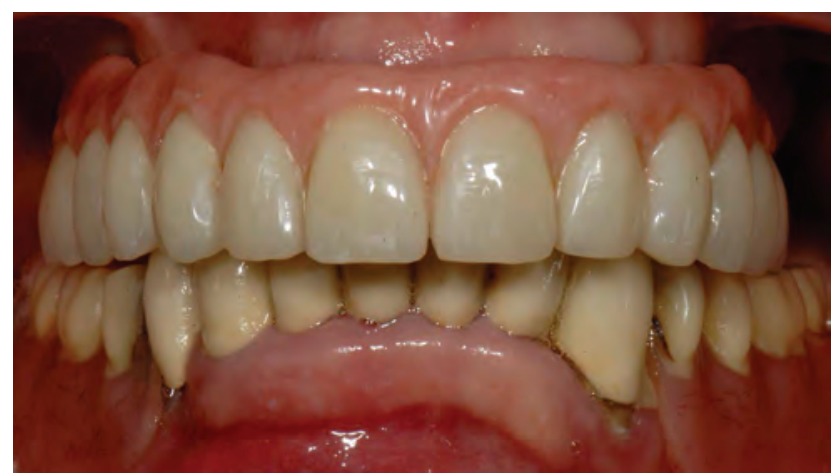

Fig. 4 Intraoral frontal view of the definitive restoration delivery.

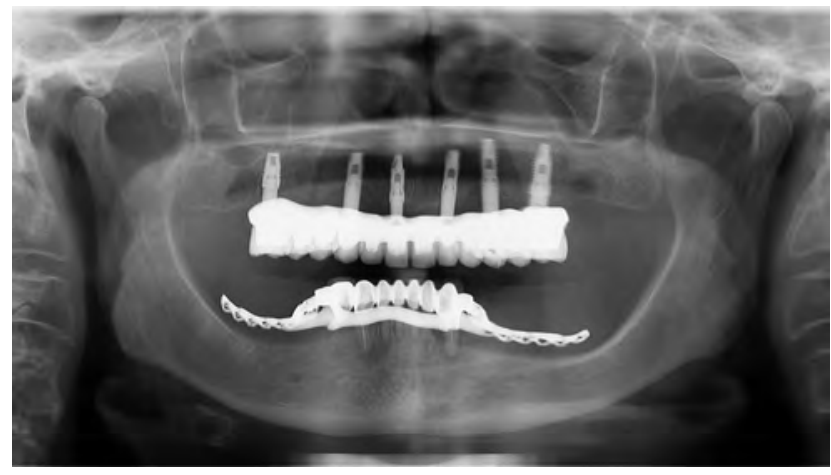

Fig. 5 Radiographic control at the definitive restoration delivery.

technique with Rinn's film holder (Rinn XCP, Dentsply, Elgin, Illinois, United States) taken at implant placement and loading (baseline) and then 1 year after. All the radiographs have been analyzed through a dedicate software (DFW2.8 for Windows, Soredex, Tuuka, Finland) and calibrated for each image using the known distance between two consecutive threads. Difference between baseline and last follow-up was taken as MBL. A dentist, not previously involved in this study, performed every radiographic measurement. 
- Bleeding index and plaque index were evaluated at six sites around each implant-abutment interface at the 1-year examination with a periodontal probe (PCPUNC156, Hu-Friedy, Milan, Italy).

- Oral health impact profile (OHIP-21) was calculated with a questionnaire of 21 questions and divided in seven subscales (functional limitations, physical pain, psychological discomfort, physical disability, psychological disability, social disability, and handicap), with two to four questions each, completed by patients. Patients were instructed to choose from five possible responses ranging from 1 (never) to 5 (very often). The questionnaire was administered by an independent dentist before treatment and yearly after definitive prosthesis delivery.

Differences between the two groups was compared with Mann-Whitney $U$ tests for continuous variables (HOIP, marginal bone levels). All the analyses were conducted at the 0.05 level of significance. Statistical analysis was performed by a software (SPSS for Mac OS X v22.o, Chicago, IL, USA).

\section{Results}

Out of 28 medical records from five centers, 14 patients (10 women and four men; range was 39-82 years old; average age was $63.6 \pm 11.7$ years) were selected according to inclusion and exclusion criteria. Of the 14 exulted patients, two did not reach the 1 -year follow-up and 12 receive mandible restorations. A total of 76 implants were inserted (range $=4-6$; average 5.4 for each patient). Two patients were light smokers. Only one patient present one systemic disease (type II diabetes). All the patient was Class II or III of Cawood and Howell. All the patients were followed for at least 1 year after function (range $=12-24$ months; average follow-up $=15.8 \pm 5.1$ months). Three out of 76 implants were connected to the prosthetic framework using only the Seeger system (3.9\%), without any screw. In four patients, the antagonist was a complete removable denture (28.6\%), setting a bilateral balanced occlusion. In the other 10 cases (71.4), natural teeth or implants were present in the antagonist arch, setting an occlusion with anterior guidance or group function (unilateral balanced occlusion). I prefer One year after loading... after loading, one implant failed (implant survival rate of $98.7 \%$ ). None of the prosthesis failed (survival rate of 100\%). One prosthetic complication was experienced in one patient. A tooth in position 21 fractured a few months after definitive loading. The prosthesis was repaired chairside in 90 minutes without any further complication.

Marginal bone level at implant placement was $0.03 \pm 0.05 \mathrm{~mm}$. Total 1 year after initial loading the marginal bone level was $0.24 \pm 0.15$. The difference was statistically significant $(0.21 \pm 0.11 \mathrm{~mm} ; p=0.000)$. I prefer One year after loading... after loading, $8.7 \%$ of the examined implant sites present positive bleeding on probing, while $6.4 \%$ of the implant sites presented plaque. The OHIP before treatment was $89.7 \pm 5.8$. I prefer One year after loading... after the treatment OHIP was $17.8 \pm 7.5$. The difference was statistically significant $(71.9 \pm 8.5 ; p=0.000)$.

\section{Discussion}

The present multicenter retrospective study was conducted to evaluate the performance of the OT Equator system (Rhein'83) used to support a screw-retained fixed dental prosthesis delivered in edentulous maxilla. To the best of the authors' knowledge, no other studies in the literature aimed to analyze OT Equator (Rhein'83) in fixed dental prosthesis. This make impossible to compare the present data with other comparable results. Even so, high implant and prosthesis survival rate were experienced after 1 year of function. Only one minor complication was experienced and easily resolved chairside. Peri-implant MBL experienced 1 year after loading was $0.21 \mathrm{~mm}$. This value is in agreement with other study evaluating one-abutment one-time concept, showing good benefits of this prosthetic protocol. Moreover, OT Equator system (Rhein'83) allows for platform switching concept helping to reduce the overall amount of peri-implant bone remodeling.

The OT Equator attachment (Rhein'83) is a global and well-established system used in the removable prosthetic protocols. ${ }^{11-15}$ Thanks to its features and great versatility, OT Equator (Rhein'83) can be used to support a screw-retained fixed dental prosthesis. Moreover, the Seeger system, represents an innovative and revolutionary acetal ring placed between the subequatorial area of the OT Equator (Rhein'83) and the cylindrical "Extragrade" abutments (Rhein'83). This new concept also allows to reduce the number of screws needed to support the prosthesis. Usually one to two screws can be avoided in case of four to six placed implants respectively, and preferable, within screwed ones. In the present study, in three out of 76 OT Equators (Rhein'83), the prosthetic screws were not used. Two out of six and one out of six implants only receive Seeger system, without screws. This feature can be successfully used to reduce anesthetic concerns caused by errors in implant positioning. ${ }^{21}$

Another benefit of the OT Bridge concept (Rhein'83) is its ability to overcoming high implant divergency even in extreme cases over 80 degree. The OT Equator system (Rhein'83) is also available as public library in most professional CAD software, allowing to plan in advance prosthetically drive implant-supported rehabilitations. ${ }^{21-23}$ These benefits may allow to integrate well-established surgical techniques, such as All-on- $4,{ }^{22}$ with promising digital technologies that allow for virtual implant planning and template-guided execution, finally improving implant accuracy and immediate loading procedures. ${ }^{2}$

Preclinical in vitro studies showed that OT Equator (Rhein'83) offered proper retention systems when supporting an overdenture. Its shape collected the strength over the head of the retainer allowing for minor stress around the peri-implant bone tissue and fixture. ${ }^{19,20,24,25}$ FEM and Von Mises analyses contribute to the development of new technologies and new materials in the biomedical field, being able to perform a large number of simulations. This allow to evaluate all the mechanical characteristics without the presence of the patient so that they can be analyzed in detail and improved where necessary, before clinical studies. ${ }^{16-18,26,27}$ 


\section{Conclusion}

The OT Equator attachment system (Rhein'83) showed successful results when used to support a fixed dental prosthesis in the maxilla screwed on four to six implants. High implant and prosthetic survival rates, very low complications, high patient satisfaction, and good biological parameters, including only $0.2 \mathrm{~mm}$ of bone remodeling were experienced 1 year after function. Further studies are needed to confirm these preliminary results.

\section{Conflict of Interest}

None declared.

\section{References}

1 Tallarico M, Meloni SM, Canullo L, Caneva M, Polizzi G. Fiveyear results of a randomized controlled trial comparing patients rehabilitated with immediately loaded maxillary cross-arch fixed dental prosthesis supported by four or six implants placed using guided surgery. Clin Implant Dent Relat Res 2016;18(5):965-972

2 Meloni SM, Tallarico M, Pisano M, Xhanari E, Canullo L. Immediate loading of fixed complete denture prosthesis supported by 4-8 implants placed using guided surgery: a 5-year prospective study on 66 patients with 356 implants. Clin Implant Dent Relat Res 2016;37(5):610-621

3 Tallarico M, Canullo L, Xhanari E, Meloni SM. Dental implants treatment outcomes in patient under active therapy with alendronate: 3-year follow-up results of a multicenter prospective observational study. Clin Oral Implants Res 2016;27(8):943-949

4 Canullo L, Tallarico M, Gracis S, Vela X, Rodríguez X, Covani U. Clinical considerations on strategies that avoid multiple connections and disconnections of implant abutments. Int J Periodontics Restorative Dent 2020;40(1):9-17

5 Tallarico M, Caneva M, Meloni SM, Xhanari E, Covani U, Canullo L. Definitive abutments placed at implant insertion and never removed: is it an effective approach? A systematic review and meta-analysis of randomized controlled trials. J Oral Maxillofac Surg 2018;76(2):316-324

6 Tallarico M, Caneva M, Baldini N, et al. Patient-centered rehabilitation of single, partial, and complete edentulism with cemented- or screw-retained fixed dental prosthesis: the first Osstem advanced dental implant research and education center consensus conference 2017. Eur J Dent 2018;12(4):617-626

7 Meloni SM, Spano G, Ceruso FM, et al. Upper jaw implant restoration on six implants with flapless guided template surgery and immediate loading: 5 years results of a prospective case series. ORAL Implantology. 2020;12(2):151-160

8 Tallarico M, Esposito M, Xhanari E, Caneva M, Meloni SM. Computer-guided vs freehand placement of immediately loaded dental implants: 5-year postloading results of a randomised controlled trial. Eur J Oral Implantol 2018;11(2):203-213

9 Tallarico M, Meloni SM. Retrospective analysis on survival rate, template-related complications, and prevalence of peri-implantitis of 694 anodized implants placed using computer-guided surgery: results between 1 and 10 years of follow-up. Int J Oral Maxillofac Implants 2017;32(5):1162-1171

10 Tallarico M, Canullo L, Caneva M, Özcan M. Microbial colonization at the implant-abutment interface and its possible influence on periimplantitis: a systematic review and meta-analysis. J Prosthodont Res 2017;61(3):233-241

11 Tallarico M, Ortensi L, Martinolli M, et al. Multicenter Retrospective Analysis of Implant Overdentures Delivered with
Different Design and Attachment Systems: Results Between One and 17 Years of Follow-Up. Dent J (Basel) 2018;6(4):71

12 Scrascia R. Martinolli M. Venezia P, Casucci A, Ortensi, L, Tallarico M. Feasibility of low profile attachments to improve quality of life on patients with implant-retained mandibular overdenture: 1-year preliminary results of a multicenter prospective case series study. J Oral Health Dental Manag 2018; 17:No. 5

13 Tallarico M, Schiappa D, Schipani F, Cocchi F, Annucci M, Xhanari E. Improved fully digital workflow to rehabilitate edentulous patient with an implant overdenture in 4 appointments: A case report. J Oral Sci Rehabil 2017;3(3):38-46

14 Xhanari E, Scrascia R, Kadiu B, Tallarico M. Two implants supporting a mandibular overdenture to rehabilitate Cawood and Howell Class V and VI patients: a proof-of-concept study. J Oral Sci Rehabil 2017;3(2):52-59

15 Tallarico M, Xhanari E, Kadiu B, Scrascia R. Implant rehabilitation of extremely atrophic mandibles (Cawood and Howell Class VI) with a fixed-removable solution supported by four implants: one-year results from a preliminary prospective case series study. J Oral Sci Rehabil 2017;3(2):32-40

16 Bramanti E, Cervino G, Lauritano F, et al. FEM and Von Mises Analysis on Prosthetic Crowns Structural Elements: Evaluation of Different Applied Materials. ScientificWorldJournal 2017;2017:1029574. doi:10.1155/2017/1029574

17 Cervino G, Fiorillo L, Arzukanyan AV, Spagnuolo G, Campagna $\mathrm{P}$, Cicciù $\mathrm{M}$. Application of bioengineering devices for stress evaluation in dentistry: the last 10 years FEM parametric analysis of outcomes and current trends. Minerva Stomatol 2020;69(1):55-62

18 Cicciù M, Bramanti E, Cecchetti F, Scappaticci L, Guglielmino E, Risitano G. FEM and Von Mises analyses of different dental implant shapes for masticatory loading distribution. Oral Implantol (Rome) 2014;7(1):1-10

19 Cicciù M, Cervino G, Milone D, Risitano G. FEM Analysis of Dental Implant-Abutment Interface Overdenture Components and Parametric Evaluation of Equator ${ }^{\circledR}$ and Locator ${ }^{\circledR}$ Prosthodontics Attachments. Materials (Basel) 2019;12(4):592. Published 2019 Feb 16. doi:10.3390/ma12040592

20 Cicciù $\mathrm{M}$, Cervino G, Bramanti E, et al. FEM analysis of mandibular prosthetic overdenture supported by dental implants: evaluation of different retention methods. Comput Math Methods Med 2015;2015:943839

21 Tallarico M, Scrascia R, Annucci M, et al. Errors in implant positioning due to lack of planning: a clinical case report of new prosthetic materials and solutions. Materials (Basel) 2020;13(8):1883

22 Soto-Penaloza D, Zaragozí-Alonso R, Penarrocha-Diago M, Penarrocha-Diago M. The all-on-four treatment concept: systematic review. J Clin Exp Dent 2017;9(3):e474-e488

23 Scrascia R, Fiorillo L, Gaita V, Secondo L, Nicita F, Cervino G. Implant-supported prosthesis for edentulous patient rehabilitation. from temporary prosthesis to definitive with a new protocol: a single case report. Prosthesis 2020;2(1):10-24

24 Cervino G, Montanari M, Santonocito D, et al. Comparison of two low-profile prosthetic retention system interfaces: preliminary data of an in vitro study. Prosthesis. 2019;1(1):54-60

25 Cicciù $\mathrm{M}$, Cervino G, Terranova $\mathrm{A}$, et al Prosthetic and mechanical parameters of the facial bone under the load of different dental implant shapes: a parametric study. Prosthesis 2019;1(1):41-53

26 Ortensi L, Vitali T, Bonfiglioli R, Grande F. New Tricks in the preparation design for prosthetic ceramic laminate veeners. Prosthesis 2019;1(1):29-40

27 Fiorillo L, D'Amico C, Turkina AY, Nicita F, Amoroso G, Risitano G, Endo and exoskeleton: new technologies on composite materials. Prosthesis 2020;2(1):1-9 